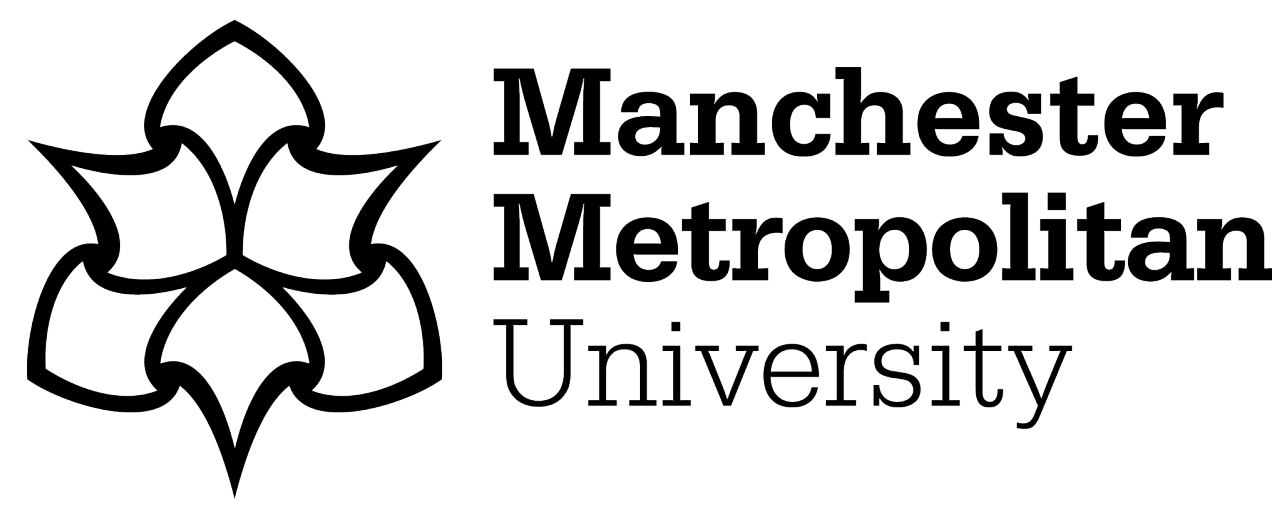

Franklin, ZC, Smith, NC and Fowler, NE (2016) A qualitative investigation of factors that matter to individuals in the pain management process. Disability and Rehabilitation, 38 (19). pp. 1934-1942. ISSN 0963-8288

Downloaded from: https://e-space.mmu.ac.uk/596498/

Version: Accepted Version

Publisher: Taylor \& Francis

DOI: https://doi.org/10.3109/09638288.2015.1107782

Please cite the published version 


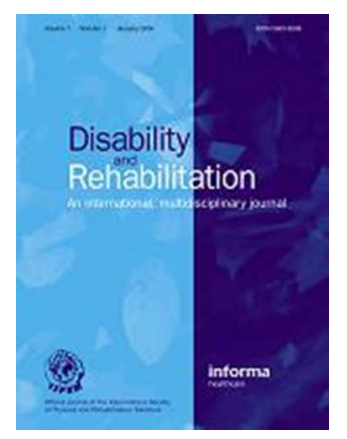

\section{A Qualitative Investigation of Factors That Matter to Individuals in the Pain Management Process.}

\begin{tabular}{|r|l|}
\hline Journal: & Disability and Rehabilitation \\
\hline Manuscript ID & TIDS-10-2014-068.R1 \\
\hline Manuscript Type: & Research Paper \\
\hline Keywords: & Chronic Pain, Pain Management, Patient Satisfaction, Patient Perspectives \\
\hline \multicolumn{2}{|c}{} \\
\hline
\end{tabular}

SCHOLARONE

Manuscripts 


\section{Implications for Rehabilitation}

- This study highlights the importance to patients of being given information to help them understand their pain condition and to learn strategies for self-management to cope better.

- Where information was not provided and inadequate time allowed for questioning and discussion, patients tended to desire more frequent access to the care services. More effective communication may result in reduced demand and more efficient management.

- Patients suggested that ongoing peer support may offer an alternative to clinician led services. 
Running Head: Qualitative Investigation of Pain Management Process

\section{Abstract}

2 Objective: Chronic musculoskeletal pain is a complex condition to manage with widespread 3 consequences including physical disability, psychosocial effects and increased use of

4 healthcare. Previous research has found patients' experiences within health care can be

5 influenced by factors such as expectations, therapist characteristics and treatment process.

6 This study used patient interviews to identify the key factors that influence individuals'

7 experiences in the management of chronic pain.

8 Design: Qualitative study using semi-structured interviews in either a home or hospital $9 \quad$ setting.

10 Methods: Semi-structured interviews were conducted on eight participants attending hospital-

11 based pain management. Participants were asked about their experiences of pain management 12 and living with a chronic pain condition. Interviews were transcribed and analysed 13 thematically.

14 Results: Three main themes were identified; impact of their condition on daily life, clinical 15 interactions and the pain management process. Understanding the condition was a key factor 16 for the patients, in particular, explanation by the clinician. The temporality of their pain 17 meant timing of appointments was critical. Patients reported factors such as family days and 18 follow up sessions would improve their treatment experience.

19 Conclusions: This study highlighted important factors for healthcare professionals and 20 patients in the management of chronic pain. Participants indicated a desire to understand their 21 condition and learn strategies for self-management to allow them to cope better. As patients 22 found benefit from being involved in the management process, discussions around the 23 options for treatment may enhance management and rehabilitation. It is important we 
Running Head: Qualitative Investigation of Pain Management Process

1 continue to research factors important to individuals with musculoskeletal pain to find an

2 effective, evidence-based framework for understanding and managing this condition.

3 Key words: Chronic Pain, Patient Satisfaction, Patient Perspectives, Pain Management

\section{$4 \quad$ Introduction}

5 Chronic musculoskeletal pain is a complex condition and one of the most significant causes

6 of suffering in the United Kingdom. The number of people being treated for/experiencing

7 chronic musculoskeletal pain is approximately 10 million and it is the second most common

8 cause for days off work (1). Due to its complexity, chronic pain is a challenging condition to

9 manage, with widespread consequences such as physical disability (2), psychosocial effects

10 (3) and regular or repeated use of healthcare services (4). There is a growing body of research

11 investigating the importance of how patients perceive their pain. Patients can find it difficult

12 to come to terms with their pain and its consequent impact on their daily life (4). This extends

13 not only to their ability to work, but also to complete normal activities of daily living (e.g.,

14 cooking, dusting, vacuuming etc.) and to interact socially with family and friends.

15 When an acute injury is experienced, pain provides a stimulus to rest and protect the

16 affected area until it heals. However, this can become maladaptive when coping with chronic

17 pain. Patients may believe that activity and movement could aggravate the injury, therefore

18 they become fearful of movement, avoid physical activity, and can become isolated, thereby

19 experiencing heightened pain and maintained disability $(5,6)$. Patient behaviour is an

20 important determinant of the maintenance and development of chronic pain and the resultant

21 disability. Patients are often referred to specialist Pain Management Programmes (PMPs) if

22 they persist in representing their case to care providers and self-report high levels of

23 disability. These programmes aim to change the way patients perceive their pain, to reduce

24 threat, promote self-management and reduce the use of health care services (2). Although

25 PMPs are designed to provide the individual with the skills to manage their pain without 
Running Head: Qualitative Investigation of Pain Management Process

1 clinician intervention, Campbell and Guy (3) found that their patient population re-engaged

2 with health care services (suggesting the PMP failed). This may be because treatment did not

3 meet patient expectations, or because they had developed an over-reliance upon healthcare

4 services to provide them with a validation of the pain experience, which may be lacking in

5 other areas of their life. Verbeek et al.(12) found that patients are normally dissatisfied with

6 treatment if it does not meet their expectations. They prefer to have a specific diagnosis,

7 instructions and information and a comprehensive plan of their treatment process. This

8 presents a significant challenge for health care providers because, for many chronic

9 conditions, no direct relationship has been established between physical pathology and pain

10 intensity. The intensity of pain, time-course, impact on daily life and personal meaning are all

11 individual experiences. Previous research has shown that patients perceive the knowledge and

12 understanding of health care providers to vary, particularly on the social and emotional

13 impact of pain (13). In addition, it has been suggested that, although clinicians adhere to the

14 current pain management guidelines, they do not always consider the patient's preferred

15 management strategies (14). If patient expectations are not met, this can promote the

16 increased use of health care services as patients seek more effective ways to cope with their

17 pain.

The majority of studies investigating PMPs have focussed on quantitative techniques to assess treatment effectiveness (4-6). However, it is important to understand how patients perceive the treatment experience and what factors contribute to their expectations and satisfaction with treatment. Previous studies have identified that relationships with health professionals, therapist characteristics, clinical outcome and treatment process (7-9) affect patient reports of satisfaction with treatment. Patients tend to judge these factors based on pre-conceived expectations of treatment and if the services they receive match such expectations. There are few studies that utilise qualitative methods to provide insight into 
Running Head: Qualitative Investigation of Pain Management Process

1 patient experiences and expectations of pain management, and the impact on their daily life

2 (10). Addressing the patient perspective is an essential step to enhance the relationship

3 between patient and clinicians leading to improved treatment outcomes and satisfaction (11).

$4 \quad$ Over the past decade, there has been an increase in research investigating patient's

5 satisfaction with treatment within the chronic pain population (15-20). Satisfaction with

6 treatment, however, is a very broad term and is not always a particularly specific measure of

7 how patients perceived their treatment process as they frequently focus solely on treatment

8 outcome. Questionnaires designed to assess satisfaction with treatment often lack specificity,

9 for example, items such as 'Are you satisfied with treatment?' are not uncommon and fail to

10 determine which aspects of the treatment (e.g. outcome, process, therapist, coping with pain

11 etc.) are the referents for patient response. Research using more qualitative methods has

12 identified key areas which patients use to evaluate their degree of satisfaction with the care

13 they have received. The findings show that relationships with health professionals, therapist

14 characteristics, clinical outcome and treatment process (9-11) are key influences on

15 satisfaction. Patients tend to judge these factors based on pre-conceived expectations of

16 treatment and if the services they receive, match such expectations. Studies have reported that

17 the therapist's ability to communicate effectively is an important factor when rating

18 satisfaction. This is particularly the case when providing a more detailed explanation for the

19 condition and providing management strategies $(19,20)$. Cooper, Smith and Hancock(21)

20 investigated physiotherapy care in chronic low back pain patients. They identified the

21 communication skills of the therapist as a key factor that also influenced other emergent

22 themes (e.g. individual care, decision-making, information, the physiotherapist and

23 organisation of care). Cooper et al.(21) highlighted the need for adapting communication

24 styles to accommodate individual needs. Participants reported feeling more involved in their 
Running Head: Qualitative Investigation of Pain Management Process

1 treatment when there was good communication compared to those who perceived

2 communication to be poor.

3 Chronic pain is a biopsychosocial problem, with each element having a distinct

4 influence upon the other. Through the use of patient interviews, the aim of this study is to

5 identify key factors which have influenced individuals' experiences in the management of

6 their chronic pain.

7

8 Method

$9 \quad$ Participants

10 Both the university ethics committee and the NHS Health Research Authority gave ethical

11 approval for this study. Participants were recruited from two hospitals and included in the

12 study if they were 18 years of age or over, had been suffering from chronic musculoskeletal

13 pain for more than three months, and had been referred to the hospital for a pain management

14 programme. Participants were invited by letter to participate in an interview. Those who

15 replied were then contacted by phone to arrange the interview. Over a three-month period, 15

16 letters were sent out and eight participants volunteered to enter the study and were

17 interviewed. It was made clear to participants at the start of the interview that their

18 involvement would have no impact on the care they received from their treating hospital and

19 they had the right to withdraw at any time. Demographic patient characteristics are presented

20 in Table 1. Of the eight participants, six were female and two male, with a mean age of 53.8

21 years. All participants had some form of chronic musculoskeletal pain, with a mean pain

22 duration of 17.3 years. All participants were either retired or unemployed due to health

23 reasons. 
Running Head: Qualitative Investigation of Pain Management Process

Insert Table 1 here.

1

2

5

6

7

8

9

10

11

12

13

14

15

16

17

18

19

20

21

22

23

24

25

26

27

28

29

30

31

32

33

34

35

36

37

38

39

40

41

42

43

44

45

46

47

48

49

50

51

52

53

54

55

56

57

58

59

60

URL: http:/mc.manuscriptcentral.com/dandr Email: davemuller@suffolk.ac.uk 
Running Head: Qualitative Investigation of Pain Management Process

1 Data collection

2 Semi-structured interviews were conducted either face-to-face $(n=2)$ or over the telephone

$3(n=6)$ depending on patient preference. An interview guide (appendix 1) was developed from

4 a literature review and the aims of the study to ensure that the same general questions were

5 covered in each interview. The interviewer, aided by the interview guide asked open-ended

6 questions to explore the key factors which had influenced each individual's experiences in

7 the management of their chronic pain. Further probe questions were asked of the participant

8 and the conversation was flexible and responsive to allow the interviewees to elaborate on

9 their responses and to gain richer information of each individual's experience (12).

10 The interviews lasted approximately 45 minutes and were audio recorded with a

11 Dictaphone and transcribed verbatim. Face-to-face interviews were conducted at the clinic

12 where the patient normally attended for treatment. Phone interviews were conducted in the

13 participant's home. Interviewing continued until saturation was reached and there was

14 repetition of the same issues.

\section{Data analysis}

16 The steps for qualitative content analysis and interpretation were guided by the thematic 17 analysis described by Braun and Clarke (13, 14). Coding and data management were 18 facilitated using qualitative data software NVivo (version 10). The first step in the analysis 19 involved initial familiarisation with the information by reading the transcript as a whole, then 20 re-reading to identify the repeated pattern of thematic content. Secondly, the presence of 21 these themes was checked across each transcript. At this point the transcripts were read 22 several times to confirm the meaning of the texts. Words and passages were highlighted and 23 assigned a code. For example, passages in which a participant described how their family 24 influences their condition were assigned the code "family support." The third step involved 
Running Head: Qualitative Investigation of Pain Management Process

1 identification of themes defined as a recurring set of codes or connection made between

2 codes. This method was chosen for systematically identifying and describing features of the

3 data which recur across participants (15). The data were returned to on several occasions to

4 define and refine the evolving themes.

5 Strategies to enhance analytic rigour included constant comparison of categories and

6 themes between the interviews and discussion of the emerging themes by the research team to

7 ensure they were comprehensive and inclusive. To eliminate bias, two of the researchers met

8 regularly to verify the data and reach a consensus. There were no differences in the themes to

9 emerge from the interviews conducted face-to-face in the clinical environment and those

10 from the home-based telephone interviews.

\section{Findings}

13 Analysis of the interviews provided three primary themes for the participants' perspective of 14 the chronic pain management process:

Theme 1: Impact on daily life; this broad theme relates to how chronic pain affects their lives on a day to day basis, subthemes include the emotional and mental impact as well as physical effects.

Theme 2: Clinical interactions; this theme related to factors about the patient's experience with the clinicians throughout the process, subthemes included communication, explanation and understanding.

Theme 3: Management process; this theme relates to positive and negative factors, which influenced their management process, subthemes included involvement, understanding, outcome and follow-up. 
Running Head: Qualitative Investigation of Pain Management Process

1 Impact on daily life

2 Participants reported a wide range of emotional and physical factors which influenced daily

3 life as a result of their chronic pain. Generally, participants found that chronic pain changed

4 their daily life significantly, more specifically, there were tasks and activities that they were

5 no longer able to complete. Participants described how the physical impact of pain affected

6 their daily lives, particularly, poor mobility, lack of sleep, fatigue, and difficulty standing or

7 sitting. All of these factors made participants feel as though they had lost their independence,

8 as others had to perform basic tasks for them.

9 “... I used to be active all the time... I know it sounds stupid, but I can't just 10 get on and do things, like house cleaning, so my Mrs. has to do it all for me, 11 and I really hate that because ya know if I see dust up on the top of the door 12 frame, I would normally just sort it out." [participant 3]

13 "my parents have to miss work to take me to an appointment." [participant 5]

Participants reported that mornings were the most difficult period of the day because of the

17 amount of time it took each participant to get ready in the morning varied, and was often dependent on the amount of sleep the night before. Depending on how they felt in the morning and how long it took to get ready, would then have an effect on the rest of the day.

“... something that does really annoy me is that they don't listen to what I say about what time of day I want my appointments because the tablets really knock me out and it makes it difficult in the mornings for me to get up in time ... they make an appointment for half eight in the morning which just doesn't help." [participant 6]

27 reporting that they had to carefully plan the day and ensure that they paced themselves in 28 order to reduce the amount of pain experienced. Participants also reported that if their pain 
Running Head: Qualitative Investigation of Pain Management Process

1 was not managed they could end up in a vicious cycle of high pain, tension and doing too

2 much. Using the coping strategies they learnt at the treatment sessions helped them avoid

3 relapses and gave more consistency to daily life.

$4 \quad$ "... you have some good days and then you try and get everything done that

5 you're trying to catch up on and then you wonder why you're in bed for a

$6 \quad$ week afterwards because you can't move." [participant 4]

and mental aspects, such as feelings of depression, lack of self-confidence and negative

mood. Participants commented on how these physical and mental aspects all contributed to

the extent that pain affected their life if they were unable to control it or implement effective

coping strategies.

"You can cope with it [the pain] a lot better if it's managed. You will never get rid of it but if it's managed you can have a reasonably normal life. But if it's not, it basically puts a full stop on it" [participant 8]

"I mean it is depressing, because it affects the quality of your life always." [participant 2] 
Running Head: Qualitative Investigation of Pain Management Process

1 "because you feel very, very alone, very alone, especially when you've got

2

3

4

$9 \quad$ with family and friends.

\section{Clinical interactions}

people in your family who don't understand and don't care and just make life really, really difficult." [participant 4]

The participants reported on a number of characteristics in relation to their experiences with clinicians and their consultation preferences. The issues reflected whether their consultants were caring/friendly, the level of communication, whether participants felt they were taken seriously about their condition and the clinician's understanding of the impact of the pain. Generally, participants liked their clinician to have a caring and friendly attitude, which gave them more confidence in the clinician's suggestions.

"I liked it because they were all so friendly and so nice and so caring and you know it was lovely." [participant 1]

Participants were more trusting and more likely to adhere to the self-management suggestions of clinicians they felt were interested in them and listened to what they had to say. Some participants, however, felt like they had to pester the clinician for an appointment or more information and that their condition was not being taken seriously. They felt at some stages of the treatment process they were passed around to different practitioners, which led to miscommunication as patients felt their treatment was not progressing. The participants understood the time restrictions that clinicians were under, however, they often felt like the 
Running Head: Qualitative Investigation of Pain Management Process

1 treatment sessions were too short and they had to push them for answers and be assertive in

2 order to make clinicians listen to them. An important factor to the participants was to have

3 more communication and contact with the clinician in order to help them better understand

4 their condition and to address issues that arose.

5 "I still feel like I'm getting passed off from one to another and no one is 6 really making a decision... they've not really said what's going to happen, it 7 just seems like we are going round in circles and I keep going back for more $8 \quad$ sessions." [participant 8$]$

Participants differed in their views of how well clinicians explained their condition

and the course of treatment that needed to be undertaken. Some participants reported that

when they were first diagnosed they were told the clinical name of their condition but not what the name actually meant or the consequences for rehabilitation or management. It was not until they did their own research and attended either a pain management or living with pain course that they truly understood the process of their pain and the consequences of their condition. There were three key areas that participants wanted more information about; (i) the problem itself, (ii) the treatment process, (iii) the long-term implications of the condition. The explanation of their condition also helped improve patient satisfaction with treatment. The level of explanation that participants received varied, the provision of some anatomical information was found to be useful, particularly when used in conjunction with pictures and drawings. In addition, this approach reassured participants that their pain was recognized as 22 real and being taken seriously. 
Running Head: Qualitative Investigation of Pain Management Process

$1 \quad$ Management process

2 Participants liked to be given an outline of what was going to happen in the management

3 process, why that particular intervention was used and how it was going to influence them.

4 Some participants became pessimistic about the treatments effectiveness.

5 "personally, I don't think it's going to do anything... because the last time I

$6 \quad$ was at physio it didn't work." [participant 3]

8 Some participants found the management process very frustrating if they could not

9 find answers for their questions, or felt as though they were just being passed from one

10 clinician to another. They also reported difficulty with travelling to appointments,

11 particularly if they have to rely on family and friends to take them. They understood that they

12 had to go to the hospital to see the clinician, however, they reported frustration if, when they

13 arrived, appointments were delayed or brief. They preferred to have more time at each

14 session in order to allow them to find out more information and gain a better understanding of

15 their course of treatment. In addition, they reported that there were very few follow-up

16 sessions to assess how the condition and the treatment were progressing and if there had been

17 any changes.

"I would like to see someone like every 3 or 4 months or something, umm just so that they can get an update, umm because I've got a load of questions that I want to ask the specialist." [participant 5]

they should be active in managing their pain, however, they reported that the treatment

24 process was often more prescriptive rather than a consultation. Participants wanted to have

25 more involvement in planning their treatment so that it addressed their individual needs and,

26 as a result, they felt they would be more likely to adhere to the advice. Benefits from the 
Running Head: Qualitative Investigation of Pain Management Process

1 PMPs were widely reported as they gave patients the opportunity to talk to people who were

2 in a similar situation to themselves. By talking with others, they were able to identify

3 different strategies to manage their pain that others had found effective. In turn, this gave

4 them more self-confidence and an empowerment to cope with their pain rather than letting it

5 control their lives. All participants reported that the coping strategies had been a key part of

6 their treatment process and would have liked to know about these strategies at an earlier stage

7 of treatment.

Participants reported that their perception of outcome changed over the course of the management process. When they first sought treatment, often their main aim was to find a cure, however, the longer the condition went on and the less effective they found the treatment, their main concern was to be better able to cope and reduce the pain.

"So I've gone from like cure/fix me to maybe, like you know, just calm it down a bit/reduce it." [participant 2]

It was important for participants to understand their condition as it changed their expectations of treatment outcome. Although the majority of participants reported that the intensity of their pain had not significantly improved, they felt better able to cope so that they had more mobility and as a result an improved quality of life. Improved daily life was important to all participants, and they reported that the coping strategies they had learnt from treatment were key to helping improve their level of activity. Participants understood they had a long-term condition that could not be cured, however, they were still willing to try anything in the hope that the treatment would fix them and they could go back to their normal life:

"You know you just have to hope that you're the lucky one and it works, but what do you do? Do you not try it because it's got a high chance it's not going to work or do you try it, on the off chance that there's a slim chance that it can like block some of the pain signals going down your 
Running Head: Qualitative Investigation of Pain Management Process

1

2

3

4

5 all participants reported their dislike of them, and how important it was that they reduced

6 their use of medication. Some participants reported that the medications worked for a while

7 but then stopped, or were ineffective on days of greater pain. Several reported that they

8 became allergic, and were afraid of the suggested side effects.

legs and get you out of some of the pain, maybe off some of your

medication, you've got to give it ago haven't you." [participant 1]

Even though prescribed medication was intended to provide relief or limit to the pain,
9

10 Discussion

11 This study provides insight into the complex nature of the impact of chronic pain on

12 participants' lives and the factors they consider to be important in the management of their these factors and their impact on the management process. The main themes which emerged were the impact on daily life, interactions with clinicians and the management process.

Although chronic musculoskeletal pain itself is not life threatening, it does significantly affect the individual's quality of life. In particular, the absence of a diagnosis and effective coping strategies can lead to life changing emotional and social consequences (16). The complex nature of chronic pain and the difficulty clinicians face in providing a clear diagnosis, present significant challenges as understanding their condition is a critical step for patients to help them engage with the treatment process. Zanini et al. (11) highlighted that enhancing the communication between patients and clinicians, by addressing factors from the patient's perspectives, helps to build a more critical discussion about the treatment options, giving the patient a greater feeling of control and higher patient satisfaction. Previous research has found that having a diagnosis for their pain is important for individuals condition. This complements previous literature by providing greater richness and detail of 
Running Head: Qualitative Investigation of Pain Management Process

1 as it gives them a feeling of legitimacy for their pain. Validation of their condition, through

2 diagnosis can help improve their social situations and patients feel less of a burden on family

3 and friends (17). Participants reported being more satisfied if their condition had been

4 effectively described to them. Particularly if diagrammes were used as this gave them a

5 greater understanding of the pain process. Without a valid explanation for their pain, patients

6 belief in the linear diagnosis-treatment-cure model was disturbed, which initially caused

7 feelings of worry, depression and anxiety about the future (3). This will in turn undermine

8 their self-efficacy with daily tasks, thus, resulting in higher disability.

The participants in this study also reported that their expectations changed over time

10 as they learnt more about the condition. In particular, as treatment progressed and the pain

11 remained, they became more interested in learning coping strategies, and identified that they

12 may have accepted the chronicity of their condition earlier if they had known more about

13 these strategies. This is particularly important for healthcare professionals to consider

14 incorporating some initial short-term coping strategies for daily tasks into the first

15 assessment. Satisfaction with treatment and expectations of outcome are key areas for

16 clinicians to consider (18). Studies have shown that patients who have remained in the care

17 system expect more information, and make higher demands on the interpersonal relationship

18 with their health care provider (18). Campbell and Guy (3) suggested that the reason some

19 patients re-present is that they are unwilling to accept the prospect of living with pain without

20 some form of input from clinicians. It could be suggested that patients who re-present for

21 treatment become attached to the support from the clinician. In relation to attachment theory,

22 Ainsworth and Bowlby (19) suggested that threats to the availability and responsiveness of

23 an attachment figure can produce dysfunctional emotional reactions. This could lead to an

24 increased attention to threatening information, which in turn reduces their self-efficacy with

25 particular tasks. These emotional reactions cause individuals to report higher levels of 
Running Head: Qualitative Investigation of Pain Management Process

1 disability and fear of movement (20), therefore, they are more likely to enter back into the

2 care system. In addition, clinicians have commented that some patients may not have a

3 willingness to self-manage and that the concept of accepting their condition is long term and

4 a 'readiness to change' is necessary before their behaviors will change (21).

5 Participants reported how beneficial it was for them to talk to other patients about

6 their pain and to discuss coping strategies with individuals in a similar situation. This gave

7 participants the opportunity to have social interactions with people who would take them

8 seriously and listen to their concerns about their condition as well as learning new strategies.

9 Such inter-patient sharing of experience and social interaction may offer an effective means

10 to engage with groups of patients and facilitate more self / group help based approaches. In so

11 doing, it may be possible to facilitate greater opportunity for patients to discuss and learn

12 about their condition without increasing the clinical resource demands of treatment.

13 Support from family and friends was important to how the patients coped with 14 exacerbations in their pain. Those who felt their family and friends understood the condition 15 and how it impacted upon their ability to complete tasks found it easier to be more active and 16 felt less isolated. On the other hand, those who had little or no family support reported feeling 17 very lonely and as though they were not needed for anything anymore. This feeling of 18 loneliness was more likely to be associated with participants becoming less active and social, 19 a loss of self-confidence and increased levels of depression. Previous research has found that 20 cognitive behavioural treatments which include an education element for family are more 21 effective at reducing pain severity and increasing activity (22). Within this study, when 22 family members had a better understanding of the patient's condition, and the variability in 23 function and experienced pain, patients felt more supported and better able to manage their 24 condition. However, participants also reported a loss of independence with daily activities, 25 which was evidenced through reliance on family members to complete daily tasks, and a role 
Running Head: Qualitative Investigation of Pain Management Process

1 reversal with their children. In line with the findings in this study, previous research has

2 identified a significant impact on the social and family roles for patients. Patients experience

3 significant reductions in parenting, participation in family and social leisure activities, and

4 role reversal (e.g. children taking their parents food shopping) (23). These factors have also

5 been associated with anxiety and worry about how the individual's pain affects their family

6 (24). It is suggested that limitations in fulfilling normal family roles intensifies negative

7 affect, and frustration of role limitations was evidenced in this study. This highlights the

8 necessity for PMPs to include an educational element for family members, as they may be

9 unaware of the impact they have on the pain-affected individual. The provision of clear

10 information about the pain process, not only to the patient but also to their immediate support

11 networks, was vital to ensuring successful management.

12 PMPs are designed to educate patients about their pain and to help them set goals

13 which will improve the areas of their lives where pain has a significant negative impact.

14 There is good evidence for the efficacy of PMPs (4-6), however, limited research has looked

15 at the impact for patients after treatment. Whilst patients reported enjoying the social

16 interactions associated with attendance on a PMP, they also indicated that at the end of the

17 management programme they went back to a feeling of isolation. Attendance at a PMP gave

18 participants something to look forward to each week, not only to have social interactions with

19 people but also to feel involved in their pain management in an active way. This finding

20 highlights the need to support follow-up sessions, which encourage individuals to be more

21 socially active and rely less on support from the clinician. This suggestion is contrary to the

22 aims of most programmes or guidelines which is to promote more self-management,

23 however, it may lead to individuals utilising less clinical appointments and lead to more

24 independence. 
Running Head: Qualitative Investigation of Pain Management Process

1 Failure to understand their condition and how best to self-manage their pain led many

2 participants to report, in the early stages of their management, that they were often 'over-

3 doing it' because they were trying to catch-up on tasks on their 'better days'. This increased

4 the likelihood of a relapse, and participants found they would then feel worse for the next

5 couple of days, both physically and mentally. In some cases, participants reported that this

6 negative reaction to activity left them worried and fearful of future activity, causing them to

7 avoid tasks due to lower self-efficacy, thereby leading to higher disability. This is typical of

8 the fear-avoidance model described elsewhere in the literature (25).

This study highlights some important implications for treatment. Firstly, the patient

10 interviews suggested that the treatment process and clinician interactions would influence the

11 individual's ability to develop effective coping strategies and their satisfaction with

12 treatment. Improving factors surrounding the treatment environment, may lead to an

13 improved patient experience. Secondly, in relation to the treatment process and clinical

14 interactions, the participants suggested that more information relating to their condition and

15 coping strategies at an earlier stage of treatment may be more beneficial. Therefore, changing

16 clinical practice guidelines to give short-term coping strategies may improve levels of

17 satisfaction. Finally, the patients within this study suggested the inclusion of a family support

18 day within PMPs would give the patients' relatives a better understanding of the effect pain

19 can have on the patient. Some hospital PMPs already include a support day, however it would

20 be beneficial to include this as an essential element. Giving relatives an active involvement in

21 the treatment programme may provide more support by relatives as they are made to feel

22 included and a sense of justification in their input. 
Running Head: Qualitative Investigation of Pain Management Process

1

2

3

4

5

6

7

8

9

10

11

12

13

14

15

16

17

18

19

20

21

22

23

24

25

26

27

28

29

30

31

32

33

34

35

36

37

38

39

40

41

42

43

44

45

46

47

\section{References}

1. Dewar AL, Gregg K, White MI, Lander J. Navigating the health care system: perceptions of patients with chronic pain. Chronic diseases in Canada. 2009;29(4):162-8.

2. Vlaeyen JW, Morley S. Cognitive-behavioral treatments for chronic pain: what works for whom? The Clinical Journal of Pain. 2005;21(1):1-8.

3. Campbell C, Guy A. Why can't they do anything for a simple back problem?A qualitative examination of expectations for low back pain treatment and outcome. Journal of health psychology. 2007;12(4):641-52.

4. Hoffman BM, Papas RK, Chatkoff DK, Kerns RD. Meta-analysis of psychological interventions for chronic low back pain. Health psychology. 2007;26(1):1.

5. Koes B, Van Tulder M, Thomas S. Diagnosis and treatment of low back pain. BMJ: British Medical Journal. 2006;332(7555):1430.

6. Williams A, Eccleston C, Morley S. Psychological therapies for the management of chronic pain (excluding headache) in adults. Cochrane Database Syst Rev. 2012;11.

7. Fitzpatrick R. Surveys of patient satisfaction. 1: Important general considerations. British Medical Journal. 1991;302:887-9.

8. Wensing M, Grol R, Smits A. Quality judgements by patients on general practice care: a literature analysis. Social Science Medicine. 1994;38:45-53.

9. Trentman TL, Cornidez EG, Wilshusen LL, Chang YHH, Seamans DP, Rosenfeld DM, et al. Patient Satisfaction in an Academic Chronic Pain Clinic. Pain Practice. 2012.

10. Bunzli S, Watkins R, Smith A, Schütze R, O'Sullivan P. Lives on hold: A qualitative synthesis exploring the experience of chronic low-back pain. The Clinical journal of pain. 2013;29(10):907-16.

11. Zanini C, Sarzi-Puttini P, Atzeni F, Di Franco M, Rubinelli S. Doctors' Insights into the Patient Perspective: A Qualitative Study in the Field of Chronic Pain. BioMed research international. 2014;2014.

12. Pope C, Mays N. Qualitative Research in Health Care. 3rd ed: Blackwell Publishing; 2006.

13. Braun V, Clarke V. Using thematic analysis in psychology. Qualitative research in psychology. 2006;3(2):77-101.

14. Guest G, MacQueen KM, Namey EE. Applied thematic analysis: Sage; 2011.

15. Marks DF, Yardley L. Research Methods for Clinical and Health Psychology. Padstow: Sage Publications; 2004.

16. Bergman AA, Matthias MS, Coffing JM, Krebs EE. Contrasting tensions between patients and PCPs in chronic pain management: A qualitative study. Pain Medicine. 2013;14(11):1689-97.

17. Toye F, Barker K. 'Could I be imagining this?'-the dialectic struggles of people with persistent unexplained back pain. Disability and rehabilitation. 2010;32(21):1722-32.

18. Verbeek J, Sengers MJ, Riemens L, Haafkens J. Patient expectations of treatment for back pain: a systematic review of qualitative and quantitative studies. Spine. 2004;29(20):2309-18.

19. Ainsworth MS, Bowlby J. An ethological approach to personality development. American psychologist. 1991;46(4):333.

20. Kobak R. The emotional dynamics of disruptions in attachment relationships: Implications for theory, research, and clinical intervention. In: Cassidy J, Shaver PR, editors. Handbook of attachment: Theory, research and clinical applications. New Your, US: Guildford Press; 1999. p. 21-43. 
Running Head: Qualitative Investigation of Pain Management Process

1 21. Gordon KaR, H., Allcock N, Bell P, Dunbar M, Gilbert S, Wallace H. Barriers to self2 management of chronic pain in primary- a research project by the charity Pain Concern. Pain 3 Nes. 2015;13(2):127-32.

4 22. Radojevic V, Nicassio PM, Weisman MH. Behavioral intervention with and without 5 family support for rheumatoid arthritis. Behavior therapy. 1993;23(1):13-30.

$623 . \quad$ Strunin L, Boden LI. Family consequences of chronic back pain. Social Science \& 7 Medicine. 2004;58(7):1385-93.

8 24. De Souza L, Frank AO. Patients' experiences of the impact of chronic back pain on 9 family life and work. Disability and rehabilitation. 2011;33(4):310-8.

10 25. Vlaeyen JW, Kole-Snijders AMJ, Boeren RGB, Van Eek H. Fear of movement/(re) 11 injury in chronic low back pain and its relation to behavioral performance. Pain. 12 1995;62(3):363-72. 
Table 1. Participant characteristics $(\mathrm{N}=8)$

\begin{tabular}{|c|c|c|c|c|c|c|c|c|}
\hline & \multicolumn{8}{|c|}{ Participant } \\
\hline & 1 & 2 & 3 & 4 & 5 & 6 & 7 & 8 \\
\hline Age & 69 & 81 & 51 & 49 & 19 & 44 & 76 & 42 \\
\hline Sex & Female & Female & Male & Female & Female & Male & Female & Female \\
\hline $\begin{array}{l}\text { Pain duration } \\
\text { (years) }\end{array}$ & 40 & 20 & 3 & 4 & 4 & 10 & 37 & 21 \\
\hline Type of pain & $\begin{array}{l}\text { Back pain } \\
\text { and arthritis }\end{array}$ & $\begin{array}{l}\text { Back pain } \\
\text { and arthritis }\end{array}$ & Low back pain & $\begin{array}{l}\text { Back pain and } \\
\text { Fibromyalgia }\end{array}$ & $\begin{array}{l}\text { Chronic } \\
\text { Fatigue } \\
\text { Syndrome }\end{array}$ & Back pain & Back pain & Back pain \\
\hline $\begin{array}{l}\text { Employment } \\
\text { status }\end{array}$ & $\begin{array}{l}\text { Retired due } \\
\text { to health } \\
\text { reasons }\end{array}$ & $\begin{array}{l}\text { Retired not } \\
\text { due to } \\
\text { health } \\
\text { reasons }\end{array}$ & $\begin{array}{l}\text { Unemployed- } \\
\text { due to health } \\
\text { reasons }\end{array}$ & $\begin{array}{l}\text { Unemployed- } \\
\text { due to health } \\
\text { reasons }\end{array}$ & $\begin{array}{l}\text { Unemployed- } \\
\text { due to health } \\
\text { reasons }\end{array}$ & $\begin{array}{l}\text { Unemployed- } \\
\text { due to health } \\
\text { reasons }\end{array}$ & $\begin{array}{l}\text { Retired not } \\
\text { due to } \\
\text { health } \\
\text { reasons }\end{array}$ & $\begin{array}{l}\text { Unemployed- } \\
\text { due to health } \\
\text { reasons }\end{array}$ \\
\hline
\end{tabular}

